\title{
Predicting the Helical Sense of Poly(phenylacetylene)s from their Electron Circular Dichroism Spectra
}

Berta Fernández, Rafael Rodríguez, Antonio Rizzo, Emilio Quiñoá, Ricardo Riguera, Félix Freire

\section{Accepted Manuscript}

\section{How to cite:}

B. Fernández, R. Rodríguez, A. Rizzo, E. Quiñoá, R. Riguera, F. Freire, Angew. Chem. Int. Ed. 2018, 57, 3666

\section{Copyright information:}

(c) 2018 Wiley-VCH Verlag GmbH \& Co. KGaA, Weinheim. This article may be used for non commercial purposes in accordance with Wiley Terms and Conditions for Use of Self-Archived Versions 


\title{
Predicting the Helical Sense of Poly(phenylacetylene)s from their Electron Circular Dichroism Spectra
}

\author{
Berta Fernández, ${ }^{*[a]}$ Rafael Rodríguez, ${ }^{[b]}$ Antonio Rizzo, ${ }^{[c]}$ Emilio Quiñoá,${ }^{[b]}$ Ricardo Riguera, ${ }^{[b]}$ and \\ Félix Freire ${ }^{\star[b]}$
}

Abstract: The calculated ECD spectrum (time-dependent density functional theory TD-DFT) for small oligomers of polyphenylacetylenes (PPAs) show a very good match with the experimental spectra of the PPA polymers, particularly with the first Cotton band associated to the helical sense of the internal polyenic backbone. This has been proven with a series of PPAs representative of cis-cisoidal, cis-transoidal, compressed and stretched polyene backbones, with identical or opposite internal/external rotational senses and allows the prediction of the helical sense of the internal helix of a PPA directly from its CD spectra.

The structural determination of helical polymers ${ }^{[1]}$ such as poly(phenylacetylene)s (PPAs) $)^{[1,2]}$ and particularly their helical sense, is a challenge with important consequences in many fields like those related to the development of new chiral materials.

Experimental methods like electron circular dichroism (ECD), $\mathrm{X}$ ray diffraction (XRD), or Atomic Force Microscopy (AFM) provide wide information on their structure, but do not determine completely the helical sense of these polymers, which are made by two coaxial helices -internal helix described by the polyene backbone and external helix described by the pendant groups[2b] For instance, CD studies provide information about the presence of a helical structure in the polyenic chain, but not the actual helical sense. For its part, images obtained from AFM show the helical sense of the external helix, but their quality is very dependent on the nature of the polymer and on the sample preparation, and in fact it is not always possible to prepare 2Dcrystals or a self-assembly monolayer to visualize its helical structure. ${ }^{[3]}$

Moreover, the two coaxial helices of a PPA can rotate either in the same or in opposite directions depending on the dihedral angle between conjugated double bonds $\left(\omega_{1}\right)$. Thus, while in ciscisoidal ( $c-c)$ PPAs internal and external helices rotate in the same direction, in a cis-transoidal (c-t) polymer both helices rotate in opposite directions. ${ }^{[2 b]}$ As a result, the information gathered from AFM (sense of the external helix) may be compatible with the two opposite senses in the internal helix leaving the structure unsolved.

[a] Rafael Rodríguez, Prof. Emilio Quiñoá, Prof. Ricardo Riguera and Prof. Félix Freire

Centro Singular de Investigación en Quimica Biolóxica e Materiais Moleculares (CIQUS) e Departamento de Quimica Orgánica Universidade de Santiago de Compostela Jenaro de la Fuente $s / n$ (Edificio CIQUS), Santiago de Compostela ricardo.riguera@usc.es, felix.freire@usc.es

[b] Prof. Berta Fernandez Departmento de Química Física

Universidade de Santiago de Compostela. 15782 Santiago de Compostela (Spain).

[c] Prof. Antonio Rizzo Fernandez Instituto per i Processi Chimico-Fisici (IPCF). Consiglio Nazionale delle Ricerche (CNR). Area della Ricerca di Pisa. I-56124 Pisa (Italy)

Supporting information for this article is given via a link at the end of the document.
This problem can sometimes be clarified by differential scanning calorimetry (DSC), ${ }^{[4]}$ where $c-c$ and $c-t$ PPAs present different thermograms, but unfortunately, the presence of peaks associated to the pendants, shifting of the polyene transitions, or just the poor definition of the thermogram, is frequent making the assignment unreliable.

Theoretical and experimental vibrational circular dichroism spectra (VCD) ${ }^{[5-7]}$ and MMFF94 energy minimization together with ECD simulation ${ }^{[8]}$ - using the time-dependent Self Consistent Field (SCF) Zerner's Intermediate Neglect of Differential Overlap (ZINDO/S) method- have also been explored as ways to solve the secondary structure of some helical polymers different from PPAs.

As for PPAs, the only paper on this topic is by Kaneko et al. that centered their study on the CD bands due to the pendants and not to those of the polyene forming the backbone, ${ }^{[9]}$ probably due to the complex structure of these polymers, made by two coaxial helices.

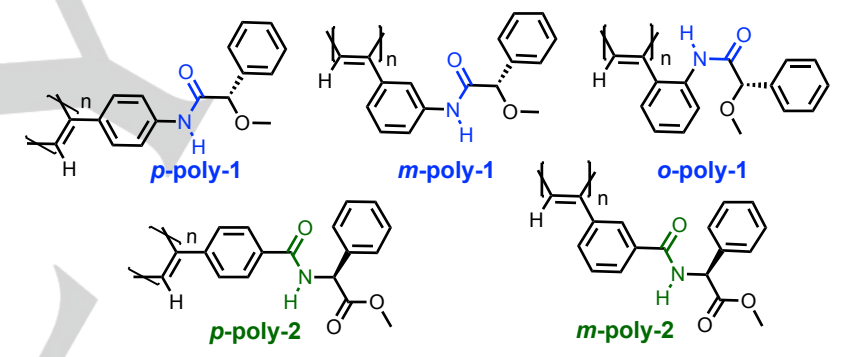

Figure 1. Polymers under study: $p$-poly-1, $m$-poly-1, o-poly-1 [anilide connection, (S)-MPA)], and $p$-poly-2, $m$-poly-2 [benzamide connection, ( $S$ )PGME].

Herein, we describe the correlation between the ECD sign of the vinylic region (first Cotton effect) of a helical PPA with the helical sense of the internal helix described by the polyene backbone. This finding is based on the coincidence between the experimental ECD spectra of a series of PPAs with well-known helical structures with the theoretically calculated ECD spectra for small oligomers.

We will show here how time-dependent DFT (TD-DFT) calculations can be used to reproduce the ECD spectra of several PPAs, possessing different left-handed, right-handed, compressed and stretched helical structures. As model compounds for this study we used two series of PPAs, namely the $p$-, $m$ - and o-ethynylanilides of (S)- $\alpha$-methoxy-a-phenylacetic acid $^{[10]}$ (MPA) and the $p$ - and $m$-ethynylbenzamides of $(S)$ phenylglycine methyl ester (PGME) [i.e., $p$-poly-1, $m$-poly-1, opoly-1, $p$-poly-2 and $m$-poly-2 respectively (Fig. 1)]. ${ }^{[10 a, 11]}$ These polymers were selected because their secondary structure is well known (AFM, SEM, CD, UV, Raman, NMR, X-ray and DSC) and cover a wide range of helical backbone possibilities [c-c, $c-t$, compressed and elongated backbones, identical or opposite internal (i) and external (e) helical senses]. 
Finally, we will also show, with examples taken from the literature, that application of this correlation allows a reliable assignment of the $P_{(\mathrm{i})}$ or $M_{(\mathrm{i})}$ sense of a PPA directly from its ECD signature.

The results obtained are summarized next, with the PPAs grouped according to their aromatic substitution pattern. For the sake of simplicity, only full discussion for $p$-substituted is described here, while $m$ - and o-substituted PPAs together with complete details on the computational approach, can be found in the SI.

$p$-Poly-1 is composed by an equilibrium mixture of helices, with the two helical senses $(P$ and $M)$ coexisting in solution. ${ }^{[10]}$ Our group previously reported a good approximation to its secondary structure based on X-ray, DSC, NMR, Raman and AFM. ${ }^{[3 a]}$ This experimentally supported structure was further refined through Molecular Mechanics, (MMFF94) $)^{[12]}$ carried out on an oligomer of $p$-poly-1 ( $\mathrm{n}=28$, monomer repeating units), folded into the $P$ and the $M$ helix.

As a result, two refined helical structures were obtained: one with the polyene backbone adopting a $c-c$ configuration, $\omega_{1}=$ $+75^{\circ}$, and a right-handed internal helix [i.e., $P_{(i)}$ ] and another one, possessing the same helical scaffold $\left(c-c, \omega_{1}=-75^{\circ}\right)$ but opposite internal helical sense [i.e., $M_{(i)}$ ].

Interestingly, when the polyene is left-handed $\left(M_{(i)}\right.$ helix, $\omega_{1}=$ $75^{\circ}$ ), the pendant groups adopt an antiperiplanar (ap) conformation between the carbonyl and the methoxy groups (Fig. $2 \mathrm{~b})$, while in the right-handed $P_{(i)}$ helical oligomer $\left(\omega_{1}=+75^{\circ}\right)$ the pendants adopt a synperiplanar conformation ( $s p$ ) (Fig. 2b).

In the two cases, the internal and the external coaxial helices, rotate in the same direction $\left[P_{(e)} / P_{(i)}\right.$ and $\left.M_{(e)} / M_{(i)}\right]$ and show three residues per turn that allow the stabilization of the scaffold by hydrogen bonds between the $\mathrm{n}^{\text {th }}$ and $(\mathrm{n}+3)^{\text {th }}$ amide groups.

Next, the ECD spectra of the two $P$ and $M$ helices were calculated.

First, DFT(rCAM-B3LYP)/3-21G calculations were performed on the $M$ helix of an $n=12$ oligomer, that was built using the dihedral angles shown in Fig. $3 \mathrm{c}$ as input and placing the pendant groups in ap conformation. A range of values between $10^{\circ}$ and $40^{\circ}$ was used in the calculations for $\omega_{3}$.

The ECD spectrum calculated in this way for the oligomer of $p$ poly-1 is shown in Fig. $3 b$ together with the experimental spectra of the polymer. The comparison shows a good match particularly in the signs of the bands of the first Cotton effect associated to the helical structure.

For a better match of the CD traces, the theoretical ECD was built from the calculated rotational strengths assuming a Gaussian band shape with a half width at half height of $0.62 \mathrm{eV}$. Also, in order to avoid overestimation of energy, ${ }^{[13]}$ the wavelength and intensity in the stationary point of the first Cotton effect were adjusted (see $\mathrm{SI}$ ).

In addition, to get a good fitting between the experimental (polymer $p$-poly-1) and the calculated (oligomer) first Cotton band, we found that the secondary structure that best reproduces the experimental ECD spectrum is the one with $\omega_{3}=$ $+36^{\circ}$ (see Fig. 3). Moreover, from the theoretical/experimental comparison studies we found that the secondary structure which posses $\omega_{3}=+10^{\circ}$ shows only partial coincidence, while other structures with different $\omega_{3}$ values provide helices that do not reproduce the experimental ECD (see SI).
Similar studies using the B3LYP functional [DFT(B3LYP)/3-21G] showed worse agreement between experimental and calculated ECD spectra, and they were not further considered (See SI). DFT(rCAM-B3LYP)/3-21G ECD studies were also carried out for the oligomer chain of $p$-poly-1 with a right-handed $P$ helix (dihedral angles shown in Fig. $3 f$ and pendant groups in $s p$ conformation), showing a good match with the experimental spectra of $p$-poly-1 (Fig. 3e).

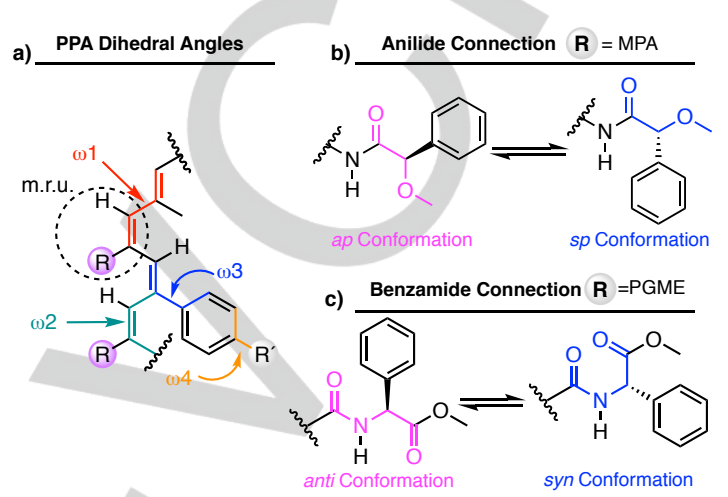

Figure 2. (a) Main dihedral angles of a poly(phenylacetylene) involved in a helical structure. Main conformers at the pendant group for (b) MPA and (c) PGME moieties.

These results on the oligomers of $p$-poly-1 revealed that the $C D$ spectra of the polymer is well reproduced by calculations on the oligomer, and that the positive $C D$ band at the vinylic region of p-poly-1 corresponds to the right $P$ sense of the polyene while the left handed sense is associated to the negative CD band.

Next, we carried out the same study on $p$-poly-2: this polymer contains the $p$-ethynylbenzamide of the (S)-phenylglycine methyl ester as pendant, and differs from p-poly-1 essentially in the connecting mode between the pendant and the backbone (a benzamide in $p$-poly-2 and an anilide in $p$-poly-1, Fig. $2 b, c) .{ }^{[10 a, 11]}$ As before, experimental data (AFM, DSC, NMR, Raman, X-Ray) were refined by MMFF94 on an oligomer affording a $c-t\left(M_{(i)}\right)$ left-handed helix with $\omega_{1}=-155^{\circ}$, with the internal and external helices rotating in opposite directions [i.e., $P_{(e)} / M_{(i)}$ ] and two residues per turn that allow further stabilization of the helical scaffold by hydrogen bonds between the $n^{\text {th }}$ and $(n+2)^{\text {nd }}$ amides, in full agreement with the experimentally known structure of the polymer.

DFT(rCAM-B3LYP)/3-21G ECD calculations on a short and representative oligomer $(n=12)$ built with the structural parameters shown in Fig. 4c, produced a theoretical spectrum that matched perfectly the experimental one obtained for $p$-poly2 once lambda values and intensities are rescaled (see Fig. 4b). Therefore, the negative sign of the first Cotton band $(380 \mathrm{~nm}$, corresponding to the HOMO $\rightarrow$ LUMO transition) of $p$-poly-2 is associated to an $M$ helix described by the polyene backbone. The agreement between the theoretical and the experimental ECD is better in the oligomer $p$-poly-2 than in p-poly-1. This is probably related to the different electrodonating/electrowithdrawing effects on the polyene of anilide ( $p$-poly-1) and benzamide ( $p$-poly-2) linkages, being the 
last one apparently better parameterized than the anilide containing $p$-poly-1.
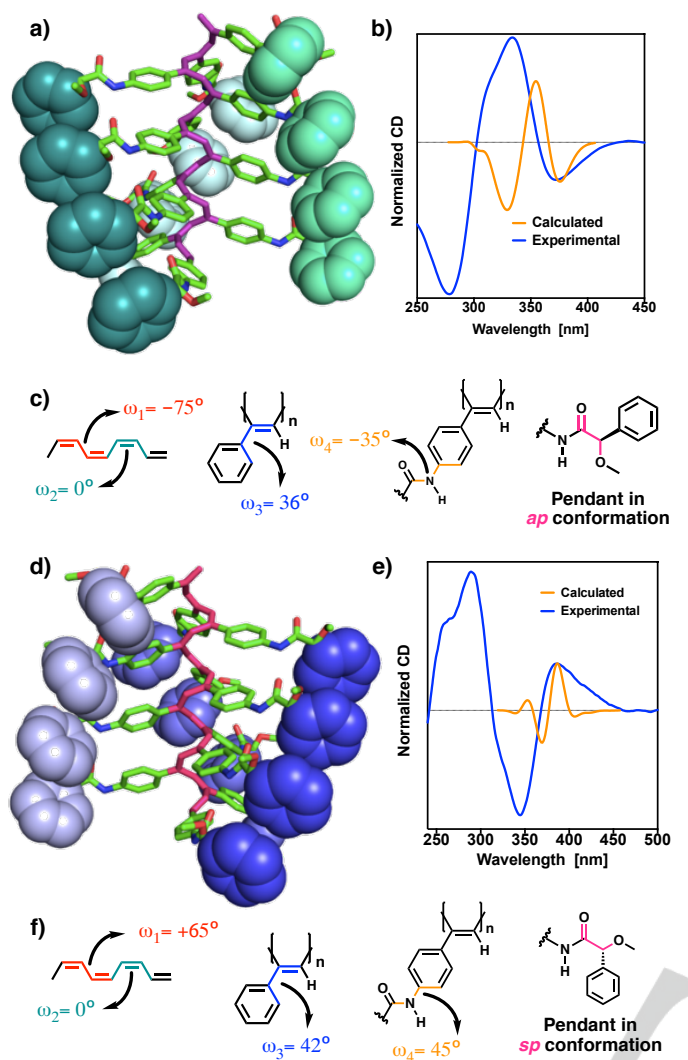

Figure 3. 3D model of the $p$-poly-1 oligomer $(n=12)$ adopting (a) an $M_{(e)} / M_{(i)}$ helix and (d) a $P_{(e)} / P_{(i)}$ helix. Dihedral angles used to build (c) and (f) oligomers of $p$-poly-1. Comparison of the theoretical and experimental CD spectra for (b) and (e) helices of $p$-poly-1 after rescaling. External phenyl groups are displayed as space-filling models.

The next families of PPAs subjected to study were the metaand ortho-substituted $m$-poly-1 [two helices, compressed (c-c) and stretched $(c-t)$ ], $m$-poly-2 [two helices, stretching degree A $(c-t)$ and stretching degree $B(c-t)$ ] and o-poly-1 [one helix, stretched, almost planar (c-t)] (Fig. 1). In all cases, DFT(rCAMB3LYP)/3-21G ECD oligomer calculations showed very good agreement with the experimental $C D$ of the polymers. Detailed description can be found in the SI.

In summary, the results presented for the polymers described in Fig. 1 show a clear correlation between the sign of the first Cotton band and the PIM sense of the internal helix:

- (a) A negative Cotton effect for the vinylic region corresponds to a polyene backbone describing an $M$ helix (left-handed).

- (b) A positive Cotton effect for the vinylic region corresponds to a polyene backbone describing a $P$ helix (right-handed).

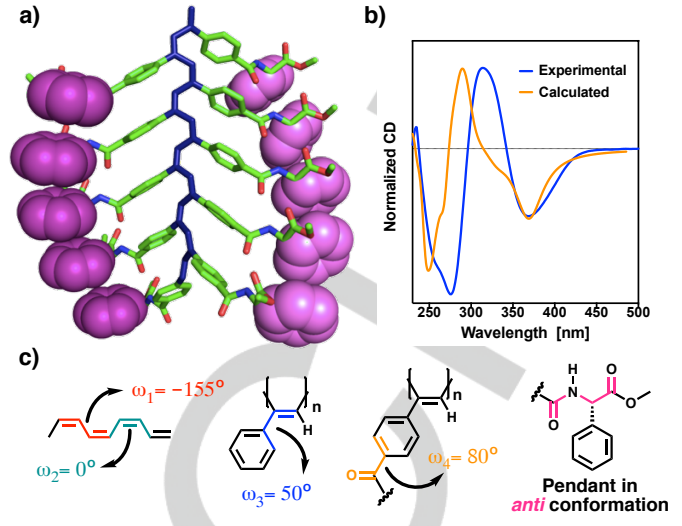

Figure 4. (a) 3D model of the $p$-poly-2 oligomer $(n=12)$ adopting a $P_{(e)} / M_{(i)}$ helix. (b) Comparison of the theoretical and the experimental CD spectra obtained in non-polar solvents for p-poly-2 after rescaling. (c) Dihedral angles used to build the structures shown.

As a test of the reliability and scope of this correlation, we applied it to some examples of PPAs taken from literature.

For instance, Tang and coworkers described a PPA with the benzamide of the $L$-Ala-OMe as pendant group (Fig. 8a). ${ }^{[14]}$ This polymer has a $c$ - $t$ backbone (DSC) and an $M$ external helix (AFM). In a $c$ - $t$ scaffold, the external and internal helices should rotate in opposite directions and therefore a $P$ helical sense was assigned to the internal helix. The polymer presents a positive Cotton band, which is in full agreement with our prediction (point b above: positive Cotton band, $P$ helical sense). Other PPAs with amino acids as pendants were reported to display $M$ external helices and positive CD. ${ }^{[15]}$ Our DSC measurements on those polymers revealed $c$ - $t$ backbones suggesting right-handed internal helices (opposite to the external ones), ${ }^{[4 a]}$ in full agreement with the $P$ helical senses predicted from the positive CD spectra.

Moreover, the application of this correlation allowed us to correct the helical structure of a PPA bearing the anilide of Mosher's acid as pendant previously assigned erroneously ${ }^{[16]}$ (see SI for a full description of this correction).

Special attention should be paid in c-c polymers because due to their geometry, very small variations in $\omega_{3}$ can lead to great changes in $\omega_{1}$ and therefore to unexpected changes in the sign of the first Cotton effect. In these cases, special care might be necessary to assign the internal helical sense.

One example of this class is represented by a PPA bearing $D$ Ala-ODec pendants ${ }^{[3 c]}$ that was shown $($ AFM) to have an $M$ external helix [i.e., $\left.M_{(\mathrm{e})}\right]$ in THF and a $P$ [i.e., $\left.P_{(\mathrm{e})}\right]$ in benzene. The polymer was considered to possess a $c$ - $t$ structure, and therefore, the internal helix in THF should rotate right-handed [i.e., $\left.P_{(i)}\right]$, while in benzene it should show a left-handed sense [i.e., $M_{(i)}$ ]. However, the experimental CD spectra showed a negative band in THF and a positive one in benzene. This CDhelical sense relationship is in apparent disagreement with our calculations but one should bear in mind that a hypsochromic shift in the UV takes place, suggesting that they could be in fact $c-c$ polyenes, where external and internal helices rotate in the same sense. 
In conclusion, in this paper we described a correlation between the sign of the CD spectra and the helical sense of the internal helix that allows the assignment of the helical sense directly from the CD spectra of the polymer. This relationship is based on the perfect match between calculated CD on short oligomers and the experimental $C D$ for polymers with different helical scaffolds (2/1 and 3/1 helices, P/M senses, and internal/external sense combinations), and was validated with examples taken from the literature.

\section{Acknowledgements}

We thank Centro de Supercomputación de Galicia (CESGA) for computational resources. We also thank the MElyC [CTQ201461470-EXP, CTQ2015-70519-P, FPI (R. Rodríguez)], XUGA (GRC2014/040, Centro singular de investigación de Galicia acreditation 2016-2019, GRC2014/040, and Axuda para Consolidación e Estruturación de Unidades de Investigación Competitivas do SUG, ED431C 2017/17) and the European Union (ERDF) for financial support.

Keywords: Helical Sense - Electron Circular Dichroism • Poly(phenylacetylene)s • Computational Study • TD-DFT

[1] a) E. Yashima, N. Ousaka, D. Taura, K. Shimomura, T. Ikai, K. Maeda, Chem. Rev. 2016, 116, 13752. b) B. A. F. Le Bailly, J. Clayden, Chem. Commun., 2016, 52, 5852. (c) B. M. Rosen, C. J. Wilson, D. A. Wilson, D. A. Peterca, M. R. Imam, V. Percec, Chem. Rev., 2009, 109, 6275; d) E. Yashima, K. Maeda, H. lida, Y Furusho, K. Nagai, Chem. Rev., 2009, 109, 6102; e) J. W. Y. Lam, B. Z. Tang, Acc. Chem. Res., 2005, 38, 745; f) J. J. L. M. Cornelissen, A. E. Rowan, R. J. M. Nolte, N. A. J. M. Sommerdijk, Chem. Rev., 2001, 101, 4039; g) D. J. Hill, M. J. Mio, R. B. Prince, T. S. Hughes, J. S. Moore, Chem. Rev., 2001, 101, 3893.

[2] a) S. Arias, F. Freire, M. Calderón, J. Bergueiro, J. Angew. Chem. Int Ed., 2017, 56, 11420; b) F. Freire, E. Quiñoá, R. Riguera, Chem. Commun., 2017, 53, 481; c) F. Freire, E. Quiñoá, R. Riguera, Chem.
Rev., 2016, 116, 1242; d) F. Freire, J. M. Seco, E. Quiñoá, R. Riguera, Adv. Polym. Sci., 2013, 262, 123.

[3] a) R. Rodríguez, J. Ignés-Mullol, F. Sagués, E. Quiñoá, R. Riguera, F. Freire, Nanoscale, 2016, 8, 3362; b) K. Okoshi, S.-I. Sakurai, J. K. Ohsawa, E. Yashima, Angew. Chem., Int. Ed., 2006, 45, 1245; c) S.-I. Sakurai, K. Okoshi, J. Kumaki, E. Yashima, , J. Am. Chem. Soc., 2006, 128,5650 .

[4] a) S. Arias, N. Nuñez-Martínez, E. Quiñoá, R. Riguera, F. Freire, Polym. Chem. 2017, 8, 3740. b) L. Liu, T. Namikoshi, Y. Zang, T. Aoki, S. Hadano, Y. Abe, I. Wasuzu, T. Tsutsuba, M. teraguchi, K. Kaneko, J. Am. Chem. Soc., 2013, 135, 602. (c) S. Leiras, F. Freire, J. M. Seco, E. Quiñoá, R. Riguera, Chem. Sci., 2013, 4, 2735.

[5] Y. Hase, K. Nagai, H. lida, K. Maeda, N. Ochi, K. Sawabe, K. Sakajiri, K. Okoshi, E. Yashima, J. Am. Chem. Soc. 2009, 131, 10719.

[6] T. Kawauchi, J. Kumaki, A. Kitaura, K. Okoshi, H. Kusanagi, K. Kobayashi, T. Sugai, H. Shinohara, E. Yashima, Angewante Chem. Int. Ed. 2008, 47, 515 .

[7] H. Z. Tang, B. M. Novak, J. He, P. L. Polavarapu, Angewante Chem. Int. Ed. 2005, 44. 7298.

[8] Y. Suzuki, J. Tabei, M. Shiotsuki, Y. Inai, F. Sanda, T. Masuda, Macromolecules 2008, 41, 1086.

[9] T. Kaneko, Y. Umeda, T. Yamamoto, M. Teraguchi, T. Aoki, Macromolecules 2005, 38, 9420.

[10] a) R. Rodríguez, E. Quiñoá, R. Riguera, F. Freire, J. Am. Chem. Soc. 2016, 138, 9620; b) F. Freire, J. M. Seco, E. Quiñoá, R. Riguera, Angew. Chem., Int. Ed. 2011, 50, 11692.

[11] I. Louzao, J. M. Seco, E. Quiñoá, R. Riguera, Angew. Chem. Int. Ed. 2010, 49, 1430 .

[12] Halgren, T. A. J. Comp. Chem., 1996, 17, 490.

[13] D. Padula, I. R. Lahoz, A. Navarro-Vázquez, C. Díaz, F. Hernández, L. Di Bari, J.-L. Alonso-Gómez, A. Rizzo, F. Santoro, M. M. Cid, Chem. Eur. J. 2015, 21, 12136.

[14] a) B. S. Shi, J. W. Y. Lam, Z. Q. Yu, B. Z. Tang, Langmuir, 2012, 28, 5770. b) K. K. L. Cheuk, B. S. Li, J. W. Y. Lam, B. Z. Tang, Macromolecules, 2008, 41, 5997.

[15] (a) K. K. L. Cheuk, J. W. Y. Lam, L. M. Lai, Y. Dong, B. Z. Tang, Macromolecules, 2003, 36, 9752; b) B. S. Li, S. Z. Kang, K. K. L. Cheuk, L. Wang, L. Ling, C. Bai, B. Z. Tang, Langmuir, 2004, 20, 7598.

[16] S. Leiras, F. Freire, J. M. Seco, E. Quiñoá, R. Riguera, Chem. Sci. 2013, 4, 2735. 


\section{Entry for the Table of Contents}

\section{COMMUNICATION}

Berta Fernández, * Rafael Rodríguez, Antonio Rizzo, Emilio Quiñoá, Ricardo Riguera, and Félix Freire*

Page No. - Page No.

Title

A computational methodology on poly(phenylacetylene) oligomers allows assigning the helical sense of polyene backbones based on their CD signature.

Predicting the Helical Sense of Poly(phenylacetylene)s from their Electron Circular Dichroism Spectra
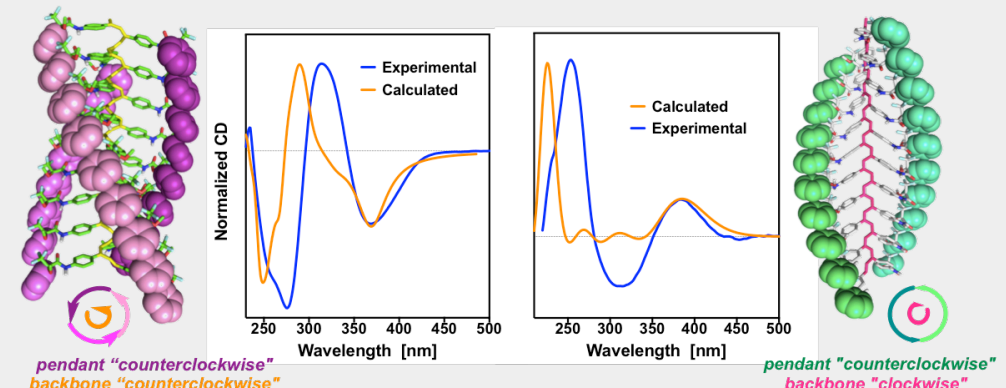